\title{
Correlation between Sulforaphane and Glutathione Peroxides-1 Accelerates Breast Cancer Cell Apoptosis
}

\author{
Roya Khaksar, Vida Firouzi, Ali Moghimpour Bijani, Elham Nozari, Ali Rostami Dehjalali, Mossa \\ Gardaneh*
}

Department of Stem Cells and Regenerative Medicine, Division of Medical Biotechnology, National Institute of Genetic Engineering and Biotechnology, Tehran/I.R. Iran

Received: February 21, 2018; Published: February 27, 2018

*Corresponding author: Mossa Gardaneh, National Institute of Genetic Engineering and Biotechnology, Pazhoohesh Blvd, Tehran-Karaj HWY Kilometer 15, PO BOX 14965/161, Tehran-Iran, Tel: (+98 21) 4458 0344; Fax: (+98 21) 4458-0395, Email: mossa65@nigeb.ac.ir, mossabenis65@gmail.com

\begin{abstract}
Induction of cell death pathways constitutes the basis of cancer chemotherapy. In this study, the co-effect of sulforaphane (SFN) and glutathione peroxidase-1 (GPX-1) on the survival of breast cancer cell line MCF-7 was examined. We produced recombinant lentivirus vectors expressing the human GPX-1 and used to transduce the MCF-7 cells. Next, we treated the cells with serial concentrations of SFN before further analyses. Cell survival was examined using the MTT viability assay. Apoptosis was measured by acridine orange/ethidium bromide co-staining and flow cytometry. Finally, changes in the expression of apoptosis and growth related molecules were detected using RT-PCR analysis. While GPX-1 over expression showed slight changes on the survival of the transduced MCF-7 cells, SFN treatment per se significantly reduced cell survival rate and this reduction correlated with increasing concentrations of SFN. By addition of SFN to GPX-1-overexpressing MCF-7 cells, survival of the cells was significantly reduced compared to untreated and SFN-treated empty vector controls. These findings were confirmed by AO/EB co-staining of the cells and flow cytometry. We also found that SFN-GPX-1 cooperation induces expression of BAX and p53 while reducing expression of BCL-2 and AKT more significantly than in SFN-treated controls. We concluded that SFN and GPX-1 can synergize to induce MCF-7 cell apoptosis.
\end{abstract}

Keywords: Breast Cancer; Mcf-7; Sulforaphane; Glutathione Peroxidase-1

\section{Introduction}

Broccoli and broccoli sprouts have long been shown to contain large amounts of glucosinolates [1]. These compounds have been shown by numerous reports to be chemo-preventive against many cancer types. Such anti-cancer activity is due to the enzymatic hydrolysis of glucosinolates from isothiocyanates [2]. Sulforaphane (SFN) is produced from glucoraphanin which is a major glucosinolate found in broccoli/broccoli sprouts [3]. SFN blocks tumor genesis primarily by inhibiting enzymes that convert procarcinogens to carcinogens and inducing enzymes that promote excretion of carcinogens [2]. Furthermore, SFN inhibits tumor cell growth by modulating cellular activities such as apoptosis or cell cycle by regulating key molecules involved in each phenomenon $[2,4]$. Other anti-tumorigenic activities of SFN include downregulation of molecules such as VEGF, HIF-1 $\alpha$, MMP-2 and MMP-9 that promote angiogenesis and metastasis [4]. SFN is also effective against cancer stem cells that rely upon given growth pathways including Wnt/ $\beta$-catenin, Hedgehog and Notch to self renews [57]. And promote cancer chemo- and radio-resistance [8-10]. SFN has been shown to inhibit breast cancer stem cells (BCSCs) in size and number by down regulating their $\mathrm{Wnt} / \beta$-catenin self-renewal pathway [11]. Glutathione peroxidase-1 (GPX-1) is a seleniumdependent antioxidant enzyme and GPX-1 gene polymorphisms and sequence alterations have been implicated in cancer development [12]. These changes minimize GPX-1 responsiveness to selenium and so reduce GPX-1 cytosolic activity ultimately leading to development of various cancers including breast cancer (BC) [12]. We have previously shown that GPX-1 can synergize with the natural turmeric compound curcumin beside anti-Her2 antibody herceptin in inducing BC cell apoptosis [13]. In this study, we examined function of GPX-1 in potentiating SFN in inducing MCF7 cell death and modifying expression levels of related molecules.

\section{Materials and Methods \\ Cell Culture and Treatment}

Human embryonic kidney cell line HEK293T and human BC cell line MCF-7 (Michigan Cancer Foundation-7) were all maintained in 
DMEM plus $10 \%$ FBS and expanded and split exactly as we have reported [13]. SFN (Sigma Aldrich, U.S.A.) was dissolved in DMSO for a stock solution of $10 \mathrm{mM}$ and frozen in aliquots at $-20^{\circ} \mathrm{C}$. Further dilutions were made in complete medium to required concentrations between 0.1-40 $\mu \mathrm{M}$ for the treatment of MCF-7 cells.

\section{Recombinant Lentivirus Production and MCF-7 Cell Infection}

We used our lent virus vector pLV-GPX1 [14]. To generate recombinant lentivirus particles harboring the human GPX-1 expression cassette beside the pLV-EGFP empty vector control [15]. The virus stocks were titrated using fresh batches of wild-type (WT) HEK293T cells and according to standard protocols [16]. For transduction purposes. MCF-7 cells were seeded in 96-well plates Table 1: Primer sequences for RT-PCR. with a number of 6000 cells/well and 24 hours later infected with pLV-GPX1 (and pLV-EGFP as a control) at a multiplicity of infection of 10 (MOI=10). We further applied the infection step to MCF-7 cells seeded in 6-well plates with a number of 3 X 105 cells/well for RNA extraction.

\section{Reverse Transcription (RT) PCR}

RT-PCR was carried out exactly as reported [15]. using primer pairs listed in (Table 1). PCR reactions were carried out for 30 cycles consisting of denaturation at $94{ }^{\circ} \mathrm{C}$ for $45^{\prime \prime}$, annealing at various temperatures (depending on primer set) for $1^{\prime}$ and extension at $72{ }^{\circ} \mathrm{C}$ for $45^{\prime \prime}$. Gel electrophoresis and band intensity measurement were duplicated as reported [15].

\begin{tabular}{|c|c|c|c|}
\hline Gene Candidate & $\begin{array}{l}\text { Primer Sequence (Forward, FW; } \\
\text { Reverse, RV) }\end{array}$ & Product Length (bp) & Reference(Accession \#) \\
\hline hGPX-1 & $\begin{array}{c}\text { FW:CTTATCGAGAATGTGGCGTCCC } \\
\text { RV:CCCACCAGGAACTTCTCAAAG }\end{array}$ & 390 & NM_000581.2 \\
\hline $\mathrm{Bcl}-2$ & $\begin{array}{l}\text { FW:GAACTGGGGGAGGATTGTGG } \\
\text { RV:GAAATCAAACAGAGGCCGCA }\end{array}$ & 211 & NM_000633.2 \\
\hline Bax & $\begin{array}{l}\text { FW: GTGGTTGGGTGAGACTCCTC } \\
\text { RV: GCAGGGTAGATGAATCGGGG }\end{array}$ & 216 & NM_004324.3 \\
\hline P53 & $\begin{array}{l}\text { FW:CCССТCCTGGCCССТGTCATCTTC } \\
\text { RV:GCAGCGCCTCACAACCTCCGTCAT }\end{array}$ & 265 & NM_001126118.1 \\
\hline Akt & $\begin{array}{c}\text { FW: GAAGGACGGGAGCAGGC } \\
\text { RV: TGTACTCCCCTCGTTTGTGC }\end{array}$ & 141 & NM_198291.1 \\
\hline -actin & $\begin{array}{l}\text { FW: AGAGCTACGAGCTGCCTGAC } \\
\text { RV: AGCACTGTGTTGGCGTACAG }\end{array}$ & 206 & X00351 \\
\hline
\end{tabular}

\section{Measurement of Intracellular GPX-1 Content}

We used an ELISA Kit (AdipoGen, San Diego, CA) to measure GPX-1 levels within each cell group. In order to calculate GPX-1 concentrations, we duplicated the procedure that we have already reported [14].

\section{Measurement of Anti-oxidant Enzymatic Activity}

The rate of NADPH oxidation is considered an activity indicator of a set of anti-oxidant peroxidases including GPX-1. NADPH oxidation was determined based on a method, reported by St. Clair and Chow [17]. that we have previously adapted and reported [15].

\section{Cell Death Analyses}

The MTT viability assay was used exactly as we have reported [18]. To measure cell viability as a percentage of untreated WT control cells.

\section{Cell Co-staining and Live Cell Count}

Since acridine orange $(\mathrm{AO})$ co-applied with ethidium bromide (EB) stains nuclear DNA green in live cells and orange in dead cells, we followed our reported co-staining method to measure the percent of viable cells [14]. Cell groups were suspended at a normal counting concentration from which $50 \mu \mathrm{L}$ was mixed with
$50 \mu \mathrm{L}$ of the stain in 96-well plates and subjected to counting under fluorescent light. Images were captured using a fluorescence microscope coupled with a Nikon digital camera. Six random microscopic fields per well were selected to count an average of 120 cells counted for each cell group in triplicates (three wells per group). Green cells and orange cells were considered, respectively, as live and dead cells. The data collected from three independent experiments were then expressed as percent of live cells by dividing the number of live cells by the number of total cells counted (live + dead). For flow cytometry, we followed our previous report [13]. To make PBS suspension of ethanol-fixed cell samples and stained them with $10 \mu \mathrm{g} / \mathrm{mL}$ propidium iodide (Sigma) before measuring the fluorescence using a FACScan (Becton Dickinson FAC Star Plus flow cytometer).

\section{Statistical Analyses}

Data in the figures are represented as the mean \pm standard error of the mean (SEM) of three or more separate experiments. Student's T-test was used to analyze differences between two groups. Differences among three or more groups were analyzed by one-way analysis of variance (ANOVA), followed by a post hoc Duncan Multiple-comparisons Test $(\mathrm{P}<0.05$, statistically significant; $\mathrm{P}<0.01$ or $\mathrm{P}<0.001$, highly significant). For correlation analyses 
(Pearson, 2-tailed), we used data of triplicates or more repeats for each set of experiment, and comparisons were made only between relevant pairs of data.

\section{Results}

\section{Successful Lentivirus Production and MCF-7 Cell Transduction}

Lentivirus construct pLV-GPX1 co-harboring GPX-1 and EGFP was used for virus production as reported previously $[14,15]$. Reporter gene expression at both post-transfection and postinfection levels indicated successful production of recombinant viruses and transduction of MCF-7 cells (Figure 1A). We expanded this transduced cell line to be able to carry out our subsequent analyses.

\section{Overexpression and Overactivity of GPX-1}

Two weeks after infection, the RNA of MCF-7 cells was extracted and subjected to RT-PCR. A 387-bp GPX-1 gene fragment was PCR amplified and resolved using agarose gel electrophoresis (Figure 1B). We found that the pLV-GPX1-infected MCF-7 cells expressed nearly threefold higher GPX-1 mRNA levels compared to the WT cells and empty vector pLV-EGFP controls (Figure 1B) $\mathrm{P}<0.01$; Duncan multiple-comparisons test). Amplification of a 206-bp
mRNA fragment from the housekeeping $\beta$-actin gene ensured equality and integrity of all RNA samples. We further measured GPX-1 protein using a GPX1-specific ELISA kit and found parallel increase in the protein levels of the enzyme in parallel with increase in its mRNA (Table 2), pLV-GPX1 compared to pLV-EGFP and WT samples, $\mathrm{P}<0.001)$. Changes in intracellular peroxidase activity were monitored by detecting NADPH oxidation. As a result of this analysis, the enzymatic activity was found induced in correlation with the levels of GPX-1 mRNA and protein (Table 3). The NADPH levels indicated the proxidase activity was over 2.5 folds up in the pLV-GPX1 cell group compared to WT and the empty vector controls (Table 3), $\mathrm{P}<0.01$, Duncan multiple-comparisons test).

Table 2: Measurement of GPX-1 protein by ELISA.

\begin{tabular}{|c|c|c|c|}
\hline Cell group & WT & pLV-EGFP & pLV-GPX1 \\
\hline GPX-1 content $(\mathrm{pg} / \mathrm{mL}$ & $46 \pm 4 \%$ & $49 \pm 7 \%$ & $111 \pm 9 \% * *$ \\
\hline
\end{tabular}

Table 3: Measurement of cell peroxidase activity.

\begin{tabular}{|c|c|c|c|}
\hline Cell group & WT & pLV-EGFP & pLV-GPX1 \\
\hline Oxidized NADPH & $5.05 \pm 0.6$ & $4.78 \pm 7 \%$ & $14.20 \pm 1.1^{* *}$ \\
\hline$(\mathrm{nmol} / \mathrm{min} / \mathrm{mg})$ & & & \\
\hline
\end{tabular}

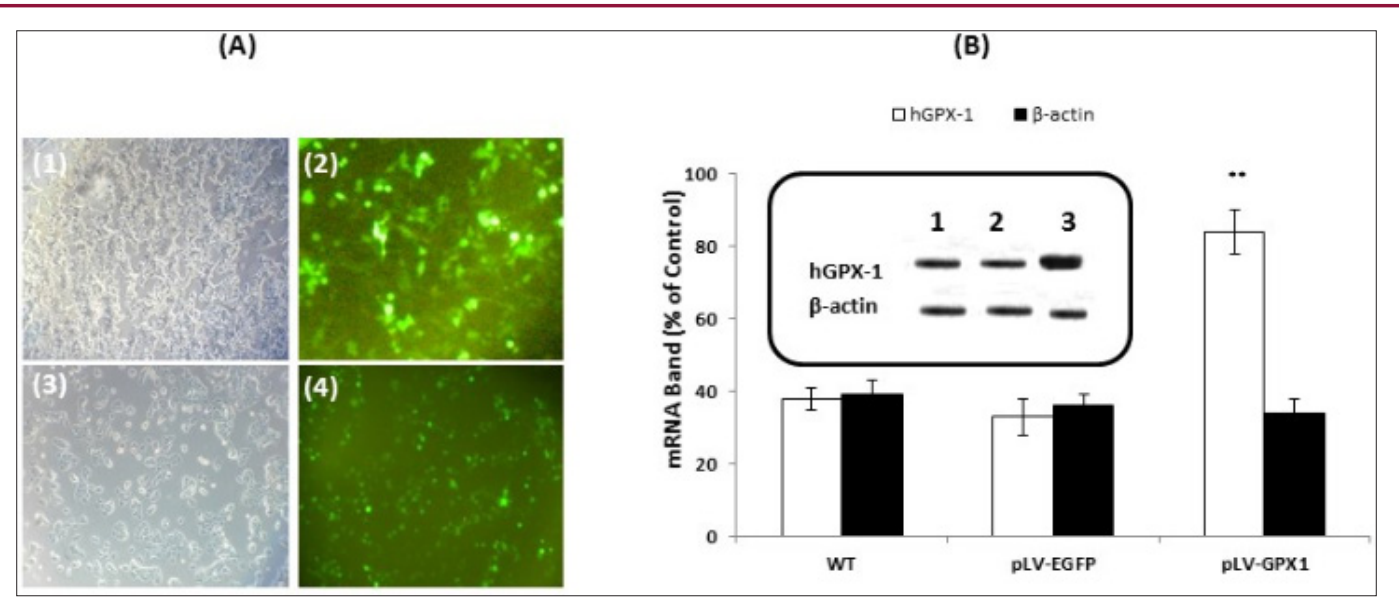

Figure 1: Expression of reporter and target genes. (A) EGFP reporter gene expression in transfected HEK-293T cells (1, 2), and in infected MCF-7 cells $(3,4)$. In order to produce recombinant viruses, HEK-293T cells were co-transfected with lentivirus packaging and envelope vectors beside pLV-GPX1 (co-harboring the EGFP expression cassette). Microscopic images of the transfected cells were taken $48 \mathrm{~h}$ posttransfection using a fluorescent microscope. The produced virus stocks were then applied to MCF-7 cells and their microscopic images were taken 2 weeks post-infection. Magnification: 100. (B) RT-PCR analysis of GPX-1 mRNA expression. The products of this analysis were resolved by agarose gel electrophoresis. The gel images of 387-bp GPX-1 sequence amplified in MCF-7 cell groups are shown. A 206-bp fragment of the $\beta$-actin cDNA was also used as an internal control. 1. Wild-type MCF-7, 2. Empty vector pLV-EGFP-infected cells, 3. pLV-GPX1-infected cells. Statistical differences in percent increase of mRNA expression between pLV-EGFP control and pLV-GPX1 cell groups are shown by **.

\section{Synergistic Effect of SFN and GPX-1 on MCF-7 Cell Apoptosis}

We first determined the LD50 for SFN by treating the wild-type MCF-7 cells with serial concentrations of the compound that stood at $24 \mathrm{M}$ (Figure 3A). We repeated the experiment for both pLVEGFP empty vector and pLV-GPX1 cell groups at MOI=10. While the empty vector controls responded to treatment similar to the WT cell groups, the pLV-GPX1 groups showed stronger response to SFN doses so that the LD50 was declined from $24 \mathrm{M}$ in controls to 14
$M$ in these cells and their survival rate was reduced significantly (Figure $3 \mathrm{~A}$ ), $\mathrm{P}<0.05$ ). Figure 3B shows images of the cells infected with a virus stock of MOI=10 and treated with $14 \mathrm{M} \mathrm{SFN}$ for $24 \mathrm{hrs}$. Next, we infected MCF-7 cells with serial MOIs of the PLV-GPX1 virus stock before treating them with $24 \mathrm{M}$ and $14 \mathrm{M}$ SFN (Figure 2A). Cell viability was declined in both groups of treated cells parallel with increase in virus concentrations (Figure 2C). For example, treatment of MCF-7 cells infected with pLV-GPX1 virus (MOI $\leq 20)$ with either SFN concentrations essentially killed nearly $70 \%$ of the 
cells compared to $50 \%$ by lower virus concentrations $(\mathrm{MOI} \leq 10)$ (Figure $2 \mathrm{~B}$ ), $\mathrm{P}<0.05$ ). As a result, the co-presence of $24 \mathrm{SFN}$ and

higher concentrations of GPX-1 severely affected the survival of MCF-7 cells by accelerating the cell death process.

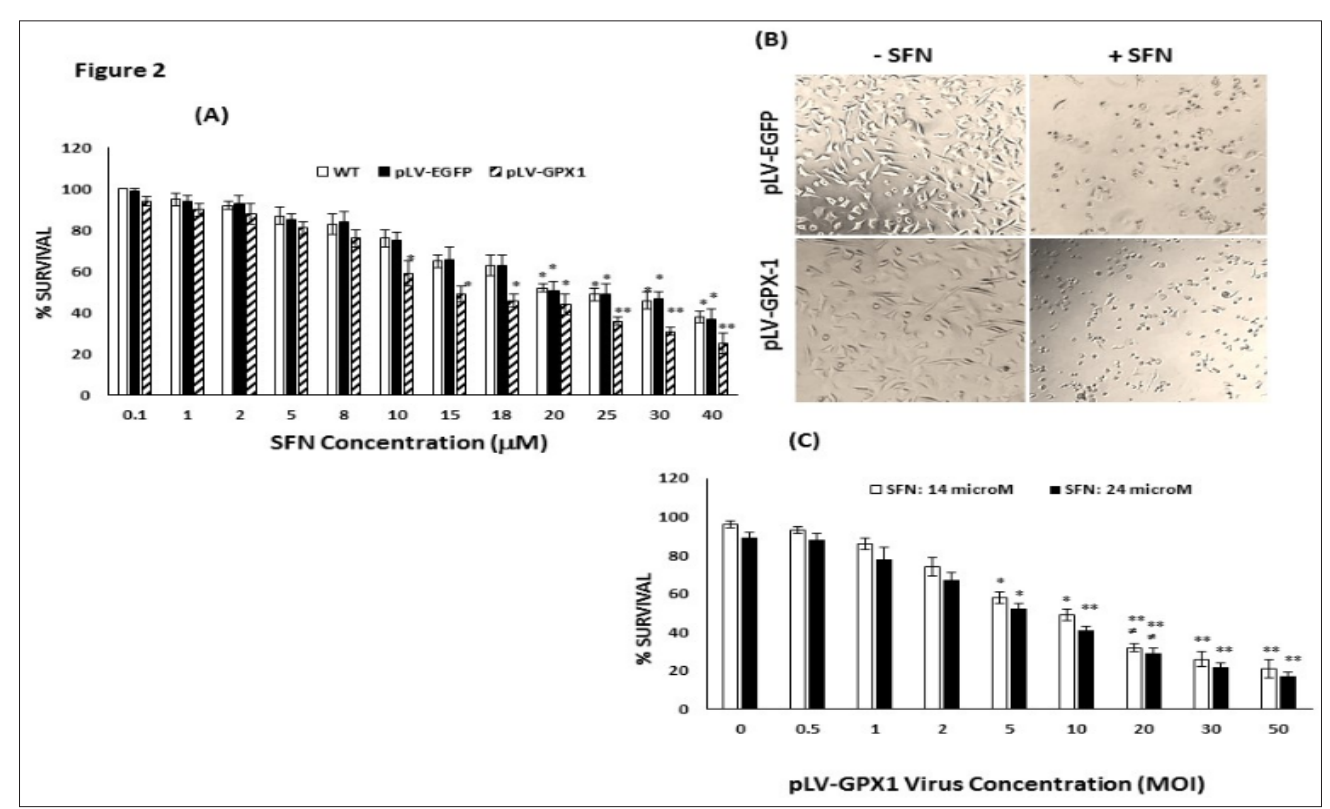

Figure 2: Treated BC cell survival. (A) Microscopic images of transduced MCF-7 cells 24 hours after treatment with sulforaphane (SFN). 1 and 2. PLV-EGFP-infected cells, untreated (1) and SFN-treated (2). 3 and 4. pLV-GPX1-infected cells, untreated (3) and SFN-treated (4). (B) Percent of survival measured by MTT assay for SNF-treated cell groups. In this and all our other graphs, each column represents an average data of three independent experiments which were carried out in triplicate. Statistical differences in survival rate between each treated cell group versus the untreated wild-type cells are shown by * or ** (C) Comparison of cell survival between two groups of cells treated with either 14 or 24 M SFN and transduced with increasing concentrations of pLV-GPX1 virus stock. Statistical differences in percent survival between each treated cell group and its untreated counterpart are shown by * or ** whereas differences between cell groups transduced with MOI=20 and their MOI=10 counterparts are shown by $\neq$.

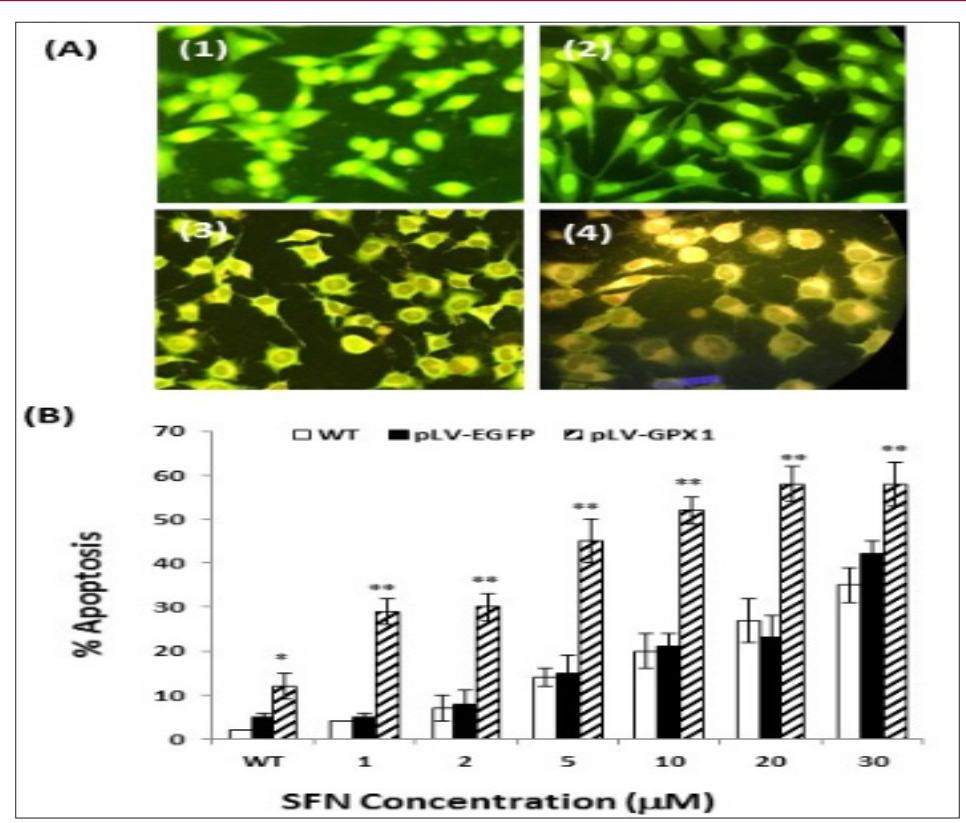

Figure 3: Quantification of apoptosis rate. (A) Microscopic images of MCF-7 cell groups using a fluorescent microscope after co-stained with acridine orange (AO) and ethidium bromide (EB). Magnification: 200x. (B) MCF-7 cell apoptosis analyzed by flow cytometry. Cell groups treated with serial concentrations of sulforaphane were analyzed by flow cytometry for their apoptosis rate. Each column in the graph represents an average data of three independent experiments. Statistical differences in apoptotic cell percentage between pLV-GPX1 and pLV-EGFP cell groups are shown by * or **. 


\section{The Apoptotic Nature of Cell Death Co-Induced by SFN and GPX-1}

We looked at the nature of cell death among our treated samples first by AO/EB co-staining and then flow cytometry both determining percent of apoptosis cells. We treated cell groups with $10 \mu \mathrm{M}$ SFN and then co-stained with AO/EB before collecting the fluorescent images. Figure $3 \mathrm{~A}$ shows the microscopic images of the co-stained cell groups and indicates that while untreated WT and pLV-EGFP cell groups were unaffected, SFN induced apoptosis in pLV-EGFP cells and accelerated cell death in pLV-GPX-1 cell groups. We quantified apoptosis among these cells by treating them with SFN. Figure 3B shows that increasing concentrations of the compound induced apoptotic cell death and this induction occurred more significantly in pLV-GPX1 cell groups compared to their WT and pLV-EGFP counterparts $(\mathrm{P}<0.05$ or 0.01$)$.

\section{Diminished Expression of Pro-Survival Genes and Increased Expression of Pro-Apoptotic Genes}

RT-PCR analysis indicated that while the expression of antiapoptotic BCL-2 and pro-survival/pro-growth genes AKT and SRC has been diminished in treated cell groups, the pro-apoptotic Bax and p53 genes have been up regulated as a result of these treatments (Figure 3). GPX-1 overexpression per se did not greatly affect apoptosis genes but treatment of WT or empty vector controls with 24 M SFN significantly reduced levels of BCL-2, AKT and SRC while significantly inducing expression of BAX and p53 towards apoptosis (Figure 3B), treated cell groups versus untreated counterparts, $\mathrm{P}<0.05$ ). This trend was accelerated when pLV-GPX1overexpressing cell groups were subjected to SFN treatment. As a result, further reduction in anti-apoptotic/pro-survival genes and conversely further increase in expression of pro-apoptotic genes were detected that were in all cases significant (Figure 3B), $\mathrm{P}<0.05$ ).

\section{Correlation between Cell Treatment and Apoptosis Rate}

Comparison between RT-PCR data, on one hand, and data of ELISA as well as enzymatic activity, on the other hand, indicated that GPX-1 mRNA levels correlated with GPX-1 protein levels and peroxidase activity, respectively, by $98 \%$ and $96 \%(\mathrm{P}<0.01 ; 2$-tailed $)$ whereas correlation between GPX-1 protein levels and its enzymatic activity stood at $95.5 \%(\mathrm{P}<0.05 ; 2$-tailed). Meanwhile, we found significant correlations between GPX-1 virus concentrations and apoptosis when the cells were treated with SFN, so that when we used GPX-1 MOI=5 and $14 \mu \mathrm{M}$ SFN, the correlation with apoptosis rate was over $96 \%(\mathrm{P}<0.01)$ whereas application of $\mathrm{MOI}=5$ or 10 with $24 \mu \mathrm{M}$ SFN caused $99 \%$ correlation with apoptosis rate $(\mathrm{P}<0.01)$ (Figure 4).

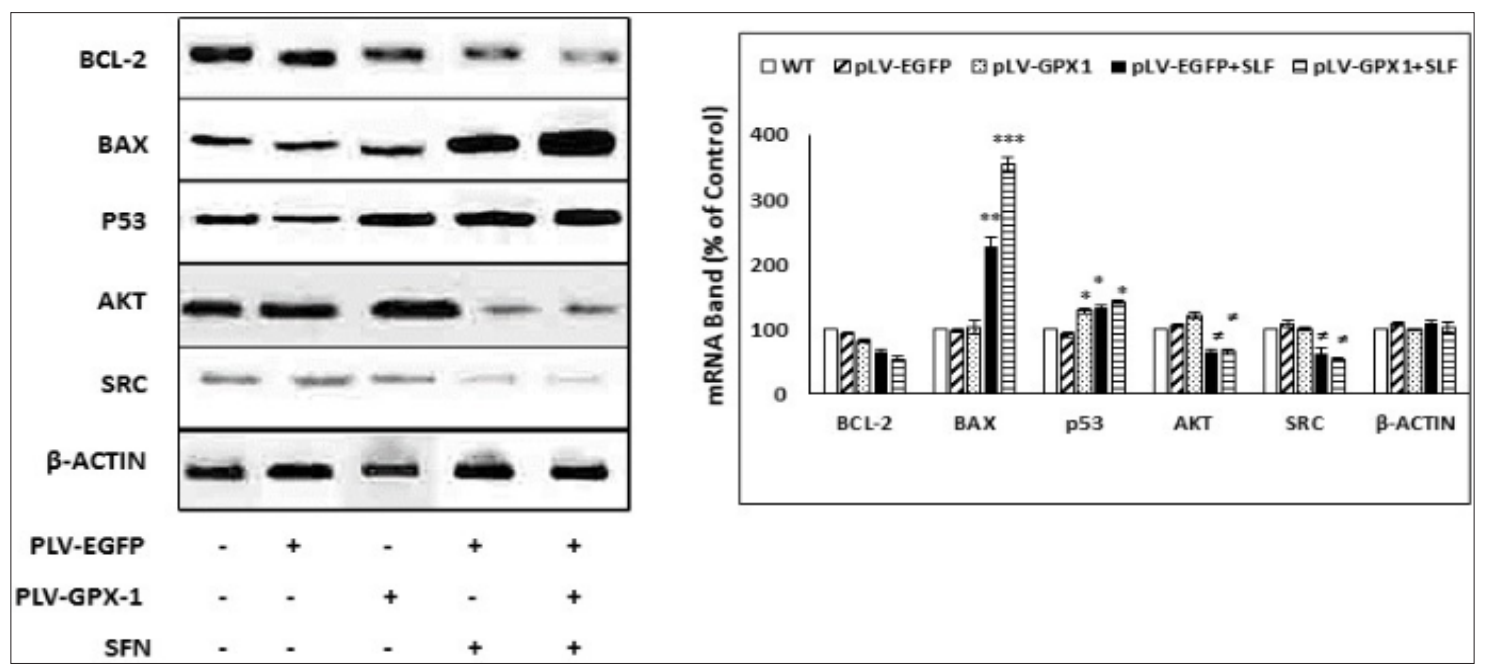

Figure 4: Changes in mRNA expression levels of pro-apoptotic and pro-survival molecules. Samples were analyzed by RT-PCR and resolved by agarose gel electrophoresis. The intensity of gel images was then analyzed using Image software (http://rsbweb.nih.gov/ij/) as we have reported (Safi 13 et al 2011). The RT-PCR analysis was repeated three times as three independent experiments. Differences of band intensity between each cell group and wild-type control are shown by * or ** for increase and by $\neq$ for reduction.

\section{Discussion}

We have previously shown that glutathione peroxidase- 1 can synergize with the natural turmeric compound curcumin beside antiHer2 antibody herceptin in inducing BC cell apoptosis [13]. In this study, we examined the co-effect of SFN and GPX-1 in inducing MCF7 cell death and modifying expression levels of related molecules. SFN per se induced apoptosis in the cells with its concentration of $24 \mu \mathrm{M}$ determined as LD50 of the drug. However in the active presence of GPX-1, this figure was dropped to $14 \mu \mathrm{M}$, a significant reduction that indicated sensitization of SFN on MCF-7 cells by
GPX-1 anti-oxidant activity. Correlation analysis indicated that GPX1 mRNA and protein significantly match with GPX-1 intracellular activity and this activity significantly sensitizes the BC cells to the pro-apoptotic effects of SFN. SFN among other nutraceuticals has drawn attention in its potential to block tumor genesis by a variety of molecular mechanisms that contribute in many key steps of tumor development [19]. Human GPX-1, on the other hand, has been shown to play important role in prevention of $\mathrm{BC}$ whereas its mutations or polymorphisms promote tumor genesis [12]. We have previously shown in SKBR-3, a HER2-amplified BC cell line, that 
GPX-1 overexpression can induce apoptosis by up regulating BAX and p53 and reducing expression of BCL-2 [13]. Our current study shows that GPX-1 per se is unable to promote these changes in MCF7 that has no HER-2 amplification and expresses moderate levels of the receptor [20]. On the other hand, MCF-7 treatment with SFN per se induces apoptosis by up regulating pro-apoptotic molecules while down regulating pro-survival genes. Furthermore, when the cells were transduced with GPX-1 lentivirus stocks and then treated with SFN, they showed significantly increased sensitivity to SFN indicating that increased activity of GPX-1 has potentiated the proapoptotic and anti-survival effects of the compound. These findings were confirmed by our correlation data.

Free radicals in the form of reactive oxygen species (ROS) play key role in initiation and progression of cancer by destabilizing cellular DNA [21]. In return, cancer cells are forced to counteract the toxic effects of ROS by adapting mechanisms for adjustment to survive that include anti-apoptotic and drug resistant measures. Furthermore, anti-oxidant enzymes including GPX-1 function against ROS by relying upon intracellular anti-oxidant defense system largely based on glutathione. In fact, GPX-1 is heavily dependent on intracellular glutathione content for its anti-oxidant function against reactive oxygen species [22]. These enzymes can affect cancer initiation and progression steps by substantially reducing intracellular ROS levels. Sulforaphane, on the other hand, accelerates cancer cell death by disrupting growth signaling pathways and inducing cell death pathways. Our findings in this study suggest that while GPX-1 reduces excessive levels of ROS that MCF-7 is exposed to upon it cancerous metabolism, in essence it facilitates the pro-apoptotic function of SFN. In order to test this hypothesis, the mechanism of GPX-1 action on MCF-7 cells needs to be studied by measuring ROS levels upon depletion of intracellular glutathione and exploring diverse molecules that play role in cancer cell metabolism including proliferation, autophagy and cancer stem cell reactions to treatment and co-treatment.

\section{Conclusion}

We showed that while GPX-1 alone is unable to produce a major shift in the cancerous state of MCF-7 cells, it potentiates the anti-survival activities of SFN. We demonstrated this change by examining cell phenotype and intracellular pathways of survival and death. The precise mechanism of SFN-GPX-1 synergy in inducing apoptosis in MCF-7 requires further investigations on cancer cell metabolism.

\section{Acknowledgement}

We thank all the staff and students in Dr. Gardaneh's bench for their support in preparing this work.

\section{Funding Sources}

This study was supported by a project from NIGEB (Grant 549)

\section{References}

1. Zhang Y, Talalay P, Cho CG, Posner GH (1992) A major inducer of anticarcinogenic protective enzymes from broccoli: isolation and elucidation of structure. Proc Natl Acad Sci USA 89: 2399-2403.
2. Clarke JD, Dashwood RH, Ho E (2008) Multi-targeted prevention of cancer by sulforaphane. Cancer Lett 269: 291-304.

3. Fahey JW, Zhang Y, Talalay P (1997) Broccoli sprouts: an exceptionally rich source of inducers of enzymes that protect against chemical carcinogens. Proc Natl Acad Sci USA 94: 10367-10372.

4. Zhang Y, Tang L (2007) Discovery and development of sulforaphane as a cancer chemopreventive phytochemical. Acta Pharmacol Sin 28: 13431354.

5. Liu S, Dontu G, Mantle ID (2006) Hedgehog signaling and Bmi-1 regulate self-renewal of normal and malignant human mammary stem cells. Cancer Res 66: 6063-6071.

6. Dontu G, Jackson KW, McNicholas E, Kawamura MJ, Abdallah WM, et al. (2004) Role of Notch signaling in cell-fate determination of human mammary stem/progenitor cells. Breast Cancer Res 6: 605-615.

7. Smalley MJ, Dale TC (1999) Wnt signalling in mammalian development and cancer. Cancer Metastasis Rev 18: 215-230.

8. Korkaya H, Paulson A, Charafe-Jauffret E (2009) Regulation of mammary stem/progenitor cells by PTEN/Akt/beta-catenin signaling. PLoS Biol 7: e1000121.

9. Shafee N, Smith CR, Wei S (2008) Cancer stem cells contribute to cisplatin resistance in Brca1/p53- mediated mouse mammary tumors. Cancer Res 68: 3243-3250.

10. Hambardzumyan D, Squatrito M, Holland EC (2006) Radiation resistance and stem-like cells in brain tumors. Cancer Cell 10: 454-456.

11. Li Y, Zhang T, Korkaya H (2010) Sulforaphane, a Dietary Component of Broccoli/Broccoli Sprouts, Inhibits Breast Cancer Stem Cells. Clinical cancer research : an official journal of the American Association for Cancer Res 16(9): 2580-2590.

12. Hu YJ, Diamond AM (2003) Role of glutathione peroxidase 1 in breast cancer, loss of heterozygosity and allelic differences in the response to selenium. Cancer Res 63: 3347-3351.

13. Gardaneh M, Shojaei S, Rahimi Shamabadi A, Akbari P (2018) Breast cancer cell apoptosis is synergistically induced by curcumin, transtuzuman and glutathione peroxidase- 1 but robustly inhibited by glial cell line-derived neurotrophic factor. Nutr Cancer 70(2): 288-296.

14. Esmaeilzadeh E, Gardaneh M, Gharib E, Sabouni F (2013) Shikonin protects dopaminergic cell line PC12 against 6-hydroxydopaminemediated neurotoxicity via both glutathione-dependent and independent pathways and by inhibiting apoptosis. Neurochem Res 38: 1590-1604.

15. Gardaneh M, Gholami M, Maghsoudi N (2011) Synergy between glutathione peroxidase-1 and astrocytic growth factors suppresses free radical generation and protects dopaminergic neurons against 6-Hydroxydopamine. Rejuv Res 14: 195-204.

16. Geraerts M, Willems S, Baekelandt V, Debyser Z, Gijsbers R (2006) Comparison of lentiviral vector titration methods. BMC Biotechnol 6: 34 .

17. St Clair DK, Chow CK (1996) Glutathione peroxidase: activity and steadystate level of mRNA. In: Punchard NA, Kelly FJ (eds) Free radicals. IRL Press, Oxford, pp227-240.

18. Gharib E, Gardaneh M, Shojaei S (2013) Upregulation of glutathione peroxidase-1 expression and activity by glial cell line-derived neurotrophic factor promotes high-level protection of PC12 cells against 6-hydroxydopamine and hydrogen peroxide toxicities. Rejuv Res 16: 185-199.

19. Gupta SC, Kim JH, Prasad S (2010) Aggarwal BB. Regulation of survival, proliferation, invasion, angiogenesis, and metastasis of tumor cells through modulation of inflammatory pathways by nutraceuticals. Cancer Metastasis Rev 29(3): 405-434.

20. Gardaneh M, Shojaei S, Kaviani A, Behnam B (2017) GDNF induces RET-SRC-HER2-dependent growth in trastuzumab-sensitive but SRC- 
independent growth in resistant breast tumor cells. Breast Cancer Res Treat 162(2): 231-241.

21. Ríos-Arrabal S, Artacho-Cordón F, León J (2012) Involvement of free radicals in breast cancer. Springerplus 2: 404.

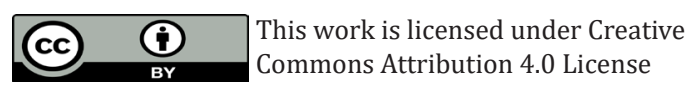

Submission Link: http://biomedres.us/submit-manuscript.php
22. Lubos E, Loscalzo J, Handy DE (2011) Glutathione peroxidase-1 in health and disease: from molecular mechanisms to therapeutic opportunities. Antioxidants \& Redox Signaling 15(7): 1957-1997.

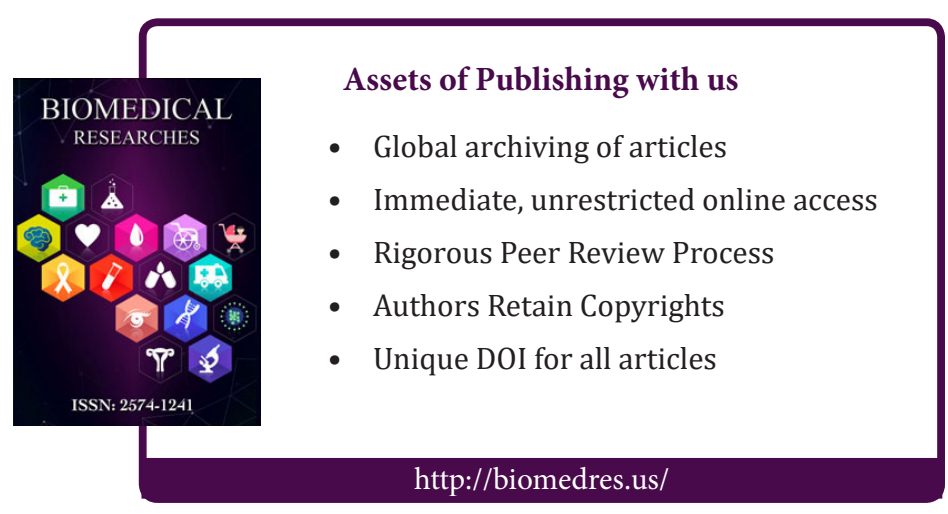

